\title{
Melanoma and Sarcoidosis in Patients Receiving or Not Antineoplastic Therapy
}

\author{
Panagiotis Gouveris a Dionysia N. Zouki ${ }^{a} \quad$ Evangelos G. Sarris ${ }^{a}$ \\ Likourgos Kolilekas $^{b} \quad$ Dimitrios Tryfonopoulos ${ }^{a} \quad$ George Papaxoinis $^{a}$ \\ Stamatina Demiri ${ }^{a}$ \\ aSecond Department of Medical Oncology, Agios Savvas Cancer Hospital, Athens, Greece; \\ b7th Respiratory Medicine Department and Asthma Center, Athens Chest Hospital "Sotiria", \\ Athens, Greece
}

\section{Keywords}

Sarcoidosis · Melanoma - Sarcoid-like reactions · BRAF/MEK inhibitors · Targeted therapy

\begin{abstract}
Sarcoidosis and sarcoid-like reactions have been associated with many solid tumors including malignant melanoma. There are reports of melanoma patients who develop sarcoidosis without having received any antineoplastic treatment, but there are also melanoma patients who have received immunotherapy or targeted therapy and, therefore, develop drug-associated sarcoidosis. Herein, we describe 2 cases of thoracic sarcoidosis which occurred in asymptomatic patients with known malignant melanoma. The first patient had metastatic disease, and she was under melanoma treatment with BRAF/MEK inhibitors at the time of sarcoidosis diagnosis. The second case involves a patient with early stage melanoma who had received no antineoplastic treatment. In both cases, the thoracic lesions were suspicious for metastatic involvement, and it was the biopsy which gave the diagnosis of granulomatous disease. Sarcoidosis induced by immune checkpoint or BRAF/MEK inhibitors seems to be more frequent in realworld studies than in large phase 3 melanoma trials. Sarcoidosis can mimic metastasis, predominately in mediastinum, representing a diagnostic pitfall. Therefore, biopsies must always be performed to exclude the metastatic spread before initiation of any antineoplastic treatment.
\end{abstract}

\section{Karger"}




\section{Introduction}

Sarcoidosis is a systemic inflammatory disease in which noncaseating granulomas develop in multiple organs. Lungs, mediastinal lymph nodes, and skin are most commonly affected. However, manifestations of sarcoidosis can also involve other organs, like salivary glands, heart, nervous system, and joints [1].

Sarcoidosis is a systemic disease. Diagnosis relies on certain criteria, including clinical and radiological presentation, histological confirmation of noncaseating granulomas, and exclusion of alternative diseases. Sarcoid-like reaction (SLR) is an alternative term, typically used in cases where we have some localized features and histologically confirmed granulomas, but the patient does not meet the criteria for the diagnosis of sarcoidosis [2].

Many solid tumors, including malignant melanoma, have been associated with sarcoidosis. Oncologic patients have an increased risk of developing sarcoidosis and vice versa. It has been reported that sarcoidosis and cancer may be diagnosed simultaneously or within a few months of one another [1].

Sarcoidosis or SLRs may be drug-related in patients under systemic antineoplastic treatment but can also be diagnosed in melanoma patients who do not receive any systemic therapy. This latter case could be described as melanoma-associated sarcoidosis [1]. Immune checkpoint as well as BRAF/MEK inhibitors may induce drug-associated sarcoidosis in melanoma patients [2].

Herein, we describe 2 cases of thoracic sarcoidosis which occurred in patients with known malignant melanoma. The first patient had metastatic disease, and she was under treatment with BRAF/MEK inhibitors at the time of sarcoidosis diagnosis. The second case refers to a patient with early stage melanoma who had received no antineoplastic treatment.

\section{Case Presentation}

\section{Case 1}

A 54-year-old female patient was diagnosed 5 years ago with superficial spreading cutaneous melanoma of anterior cervical region, Clark V, mitotic rate $>5 / \mathrm{mm}^{2}$, with clinically positive cervical lymph nodes and multiple secondary liver lesions. The melanoma proved to be BRAF-mutated. Due to a delay in the approval of targeted treatment by the local authorities, the patient received 4 cycles of chemotherapy with dacarbazine, with complete response in liver metastases, and progressive disease in cervical lymph nodes.

The patient started treatment with the combination of vemurafenib/cobimetinib. A few months later partial response in the cervical lymph nodes was noted, while she was still under targeted therapy. Two years after vemurafenib/cobimetinib initiation and with the patient being asymptomatic, computed tomography (CT scan) of chest revealed multiple suspicious mediastinal and hilar lymph nodes (shown in Fig. 1). Moreover, fluorodeoxyglucose (FDG)positron emission tomography (PET) CT scan was performed. It confirmed pathologic mediastinal lymph nodes with SUV max: 4. Accordingly, bronchoscopy and endobronchial ultrasound-guided transbronchial needle biopsy were performed. Histologic examination revealed non-necrotizing sarcoid type epithelioid cell granulomas. Angiotensin-converting enzyme (ACE) value was elevated (87 U/L). Treatment with hydroxychloroquine $200 \mathrm{mg}$ once a day and prednisolone $5 \mathrm{mg} 3$ times a day was decided. Six months later, CT scan was negative for mediastinal lymph nodes, and hydroxychloroquine was interrupted. PET-CT scan was repeated at about 12 months from the diagnosis of sarcoidosis. It was also negative for mediastinal nodes, and in general negative for progressive disease regarding melanoma. ACE value was within normal limits (48 U/L). The patient never stopped treatment with vemurafenib/cobimetinib.

\section{Karger'}


Fig. 1. Chest CT scan - Case 1. The arrow shows enlarged mediastinal lymph nodes. CT, computed tomography.
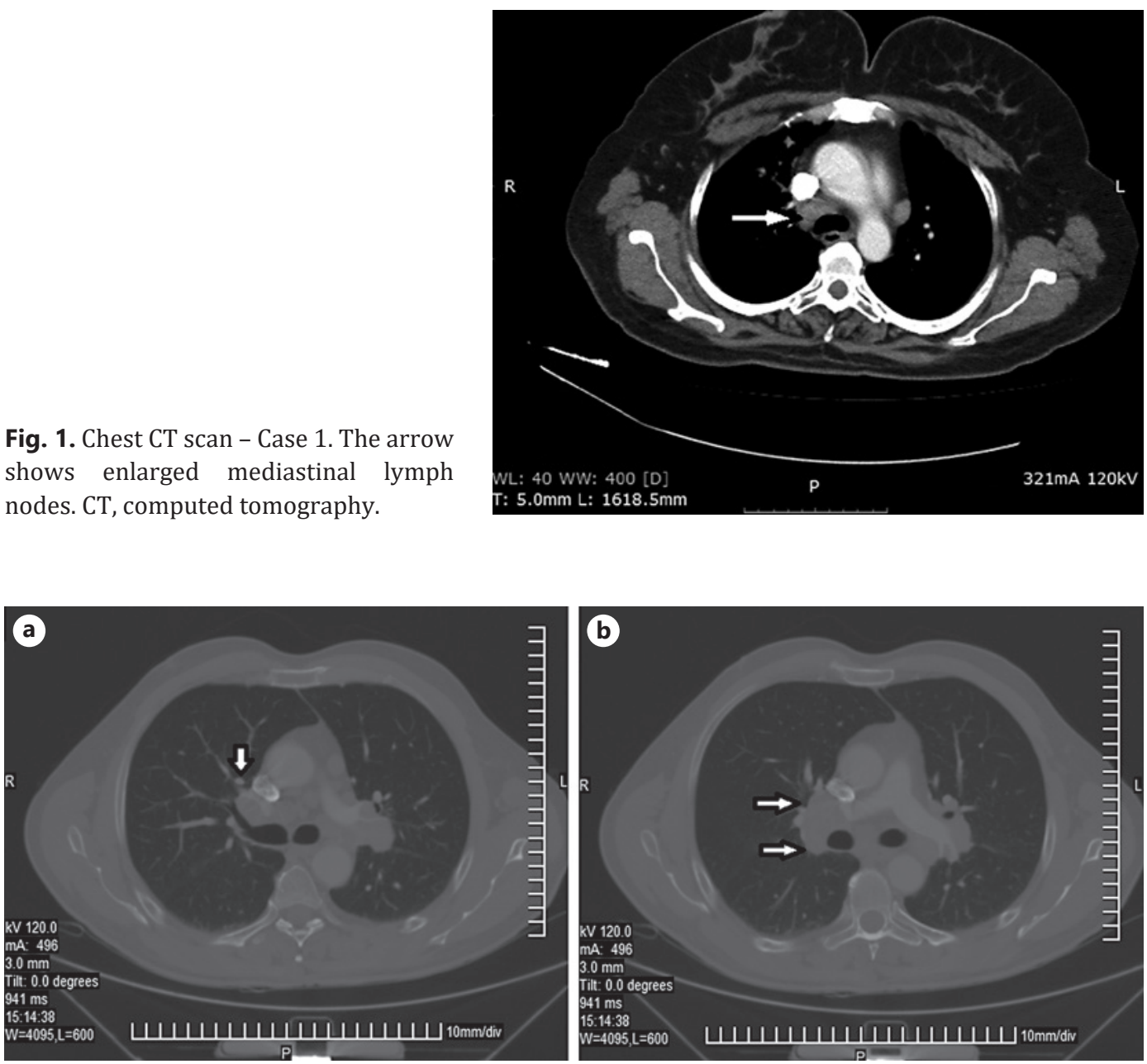

Fig. 2. Chest CT scan - Case 2. a The arrow indicates 1 pulmonary nodule. $\mathbf{b}$ The arrows show enlarged mediastinal lymph nodes. CT, computed tomography.

She is still receiving a maintenance dose of prednisolone $5 \mathrm{mg}$ once a day, and till now, she continues being free from malignant melanoma progression.

Case 2

A 60-year-old male patient was diagnosed 3 years ago with superficial spreading cutaneous melanoma of back, mitotic rate $7 / \mathrm{mm}^{2}$, Clark IV, Breslow $1.35 \mathrm{~mm}, \mathrm{~T} 2 \mathrm{aN0M0}$, stage IB. Three years later, and with the patient being asymptomatic, chest CT scan revealed 2 pulmonary nodules (shown in Fig. 2a), and multiple bilateral mediastinal (shown in Fig. 2b) and hilar lymph nodes up to $3 \mathrm{~cm}$ in diameter. Tuberculosis was excluded, and bronchoscopy and endobronchial ultrasound-guided transbronchial needle biopsy were performed. Histologic examination revealed sarcoidosis. ACE value was elevated (78.6 U/L). Four months after the initial diagnosis of sarcoidosis, chest computer tomography revealed a slight decrease in hilar lymph node size $(2.7 \mathrm{~cm}$ from $3 \mathrm{~cm})$. ACE value was almost stable $(84.5 \mathrm{U} / \mathrm{L})$. The patient remains under close follow-up, and he is free from malignant melanoma progression. He does not receive any treatment for sarcoidosis.

From January 2015 until July 2020,133 patients with malignant melanoma were admitted to our Oncology Clinic. Sarcoidosis was diagnosed in 2 of them (case 1 and case 2), which 
accounts for $1.5 \%$ of the studied population. A total of 24 patients were receiving targeted therapy with BRAF/MEK inhibitors, but only one patient (case 1,4\%) developed sarcoidosis. Among patients who were under treatment with checkpoint inhibitors, no case of sarcoidosis was denoted. Of 85 patients with early stage melanoma who did not receive checkpoint inhibitor or targeted treatment, 1 patient (case 2, 1\%) developed sarcoidosis.

\section{Discussion}

Thoracic metastases are very common in metastatic melanoma. They may appear as lung nodules, which is the most frequent pattern, but also as hilar or mediastinal lymph nodes [1, 3]. Therefore, a suspicious thoracic lesion in melanoma patients has a strong possibility to be melanoma recurrence or progression. Thoracic sarcoidosis has been described as a great mimicker or pretender and may imitate metastatic disease. The thoracic lesions in our 2 melanoma patients could represent metastases. PET-CT scan is a very useful diagnostic tool in the identification of metastatic malignant lesions. However, it may be difficult to distinguish metastatic infiltration and sarcoidosis because a moderately elevated fluorodeoxyglucose uptake can be detected in malignancies as well as in an inflammatory situation. Even in PET-CT scan, sarcoidosis can still mimic cancer recurrence or metastatic disease progression [4-6].

Therefore, if we have a melanoma patient with suspicious new thoracic lesions, it is necessary to perform a biopsy to exclude metastasis which is the most life-threatening and the most frequent scenario. However, there is still a possibility for the lesions to be benign, including the possibility of sarcoidosis.

Notably, coexistence of metastasis and sarcoidosis within the same anatomical area has been described. For example, metastatic lesions may coexist next to lymph nodes with sarcoidlike features [6]. There is also a report of melanoma lymph node metastasis occurring concurrently with sarcoidosis, affecting lymph nodes, and the lungs [7]. The risk of simultaneous metastasis in sarcoidosis lesions must be considered by clinical practitioners. Such cases may constitute a diagnostic pitfall.

Regarding melanoma-associated sarcoidosis, a common etiologic factor between the 2 entities seems to exist. Ultraviolet light is a well-known etiologic factor for melanoma. However, it may also contribute to the pathogenesis of sarcoidosis, via upregulation of tumor necrosis factor-alpha (TNF- $\alpha$ ), which induces granuloma formation $[1,8]$. When it comes to drug-associated sarcoidosis, there are many reports of sarcoidosis related to immune checkpoint inhibitors, while there are also reports of SLRs related to targeted therapy [2].

Most of the published cases of sarcoidosis in patients with melanoma have been associated with immunotherapy [2]. This is inconsistent with our study, as none of our patients under immune checkpoint blockade developed sarcoidosis. Monoclonal antibodies targeting cytotoxic T-lymphocyte-associated protein 4 and programmed cell death protein 1 (PD-1) or its ligand are drugs that cause the "famous" immune-related adverse events, such as colitis and pneumonitis. It has been shown that the cytotoxic T-lymphocyte-associated protein 4 blockade results in an increase in Th-17 CD4+ cells in peripheral blood, which induces an extended production of pro-inflammatory molecules, like IL-6 and TNF- $\alpha$ [2, 9]. These cytokines may contribute to sarcoidosis development. Though less clear, it is believed that PD-1 inhibition may also induce pro-inflammatory Th-1 and Th-17 immune responses and subsequent T-cell proliferation in various tissues, including lungs and lymph nodes. It has also been shown that PD-1 pathway is upregulated in sarcoidosis and that blockade of this signaling axis restores sarcoidosis CD4(+) T-cell impaired proliferative capacity $[2,10]$.

The highest incidence rate of sarcoidosis or SLRs in the context of checkpoint inhibitors ever reported was $22 \%$ (10 out 45 patients) and comes from a retrospective study with adjuvant immu-

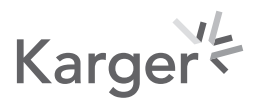


notherapy [3]. In a phase 2 neoadjuvant melanoma trial with combination of ipilimumab and nivolumab, $5.8 \%$ of patients $(n=86)$ developed SLRs, which is also a relatively high incidence [11].

Focusing on famous phase 3 trials of immunotherapy in melanoma we have to observe that sarcoidosis has never been referred as an adverse event of nivolumab, pembrolizumab, or ipilimumab, with just one exception: in Keynote 054 double-blinded phase 3 trial (of pembrolizumab vs. placebo after complete resection of stage III melanoma), sarcoidosis was diagnosed in $1.4 \%$ of patients receiving pembrolizumab $(N=509)$. All cases were of grade 1 or 2. There was no case of sarcoidosis in the placebo arm $(N=502)$ [12].

BRAF and MEK inhibitors have been associated with a variety of skin adverse events, such as granulomatous eruptions, panniculitis, and second primary cutaneous malignancies. Development of SLRs seems to be a rather paradoxical adverse event of these drugs. However, there are data confirming the immunomodulatory effect of these drugs on the tumor microenvironment. Inhibition of the mitogen-activated protein kinase pathway has been related with increased CD8+ T-cell infiltration and programmed cell death-ligand protein 1 expression. Pathogenesis of the SLRs could be further explained by increased levels of TNF- $\alpha$ and IFN- $\gamma$, which can induce granuloma formation $[2,13]$.

Therefore, regarding SLRs in melanoma patients receiving BRAF/MEK inhibitors, we cannot exactly know the extent of contribution for melanoma itself or for melanoma treatment. However, it is difficult to ignore a possible causal role of BRAF/MEK inhibitors.

There are no reports of BRAF/MEK inhibitors-related sarcoidosis in the great phase 3 trials of targeted melanoma treatment. However, there are reports of such reactions in realworld studies $[1,2,13,14]$. Thus, association of BRAF/MEK inhibitors with SLRs is less established than the one with checkpoint inhibitors, but it cannot be ignored.

In our first case, the interval between vemurafenib initiation and the occurrence of sarcoidosis was 2 years. She represented the only case of sarcoidosis out of 24 patients who received targeted therapy (prevalence 4\%). In a French study, there were 5 cases of sarcoidosis, most of them with cutaneous involvement, out of 70 melanoma patients treated with vemurafenib (prevalence 7.1\%). The mean time between vemurafenib introduction and the onset of sarcoidosis was 6 months. There was 1 case of thoracic sarcoidosis, where the interval was just 2 months [13]. Interestingly, in our case report, the female patient under vemurafenib treatment had partial response regarding her melanoma. Withdrawal of vemurafenib was not required and the patient maintains till now the partial response. This outcome is consistent with the French study, where most patients developing sarcoidosis continued vemurafenib and had good outcomes regarding melanoma [13]. Regarding our second case of melanomaassociated sarcoidosis, the interval between the diagnosis of melanoma and sarcoidosis is 3 years, which is consistent with the estimations of the previous studies $[1,15]$.

In the greatest study of sarcoidosis in melanoma individuals, which actually included a huge number of 1,199 patients, prevalence of sarcoidosis was somewhat low (0.58\%) [15]. Of course, at that time we did not have the data we have now from anti-PD-1 treatment and BRAF/MEK inhibitors. Such data have been included in more recent studies [1-3, 11-14].

\section{Conclusion}

Sarcoidosis may be diagnosed in melanoma patients in the absence of any antineoplastic treatment, but it may especially occur during or after treatment with immunotherapy or targeted therapy. It seems to be more frequent in real-world studies than in large phase 3 melanoma trials. What is more important, sarcoidosis can mimic metastatic disease, predominately in mediastinum, and for this reason, it can represent a diagnostic pitfall. Therefore, biopsies must always be performed before initiation of any antineoplastic treatment.

\section{Karger'}




\section{Case Reports in Oncology}

Case Rep Oncol 2021;14:1059-1065

\begin{tabular}{l|l}
\hline DOI: $10.1159 / 000516035$ & (c) 2021 The Author(s). Published by S. Karger AG, Basel
\end{tabular} www.karger.com/cro

Gouveris et al.: Melanoma and Sarcoidosis in Patients Receiving or Not Antineoplastic Therapy

\section{Statement of Ethics}

Ethics approval by committee was not required for this case report. It was conducted in accordance with the 1964 Helsinki Declaration and its later amendments or comparable ethical standards. Written informed consent was obtained from the patients for publication of this case report and any accompanying images. A copy of the written consent is available for review by the Editor of this journal.

\section{Conflict of Interest Statement}

The authors have no conflicts of interest to declare.

\section{Funding Sources}

No funding was received for the preparation of this manuscript.

\section{Author Contributions}

Conceptualization: P.G. and S.D. Data curation: P.G. and D.N.Z. Investigation, Methodology: D.N.Z. and E.G.S. Writing - original draft: P.G., D.N.Z., L.K., and G.P. Writing - review and editing: D.T. and S.D. All the authors have read and approved the manuscript.

\section{References}

1 Beutler BD, Cohen PR. Sarcoidosis in melanoma patients: case report and literature review. Cancers. 2015 Jun 15;7(2):1005-21.

2 Dimitriou F, Frauchiger AL, Urosevic-Maiwald M, Naegeli MC, Goldinger SM, Barysch M, et al. Sarcoid-like reactions in patients receiving modern melanoma treatment. Melanoma Res. 2018 Jun;28(3):230-6.

3 Chorti E, Kanaki T, Zimmer L, Hadaschik E, Ugurel S, Gratsias E, et al. Drug-induced sarcoidosis-like reaction in adjuvant immunotherapy: increased rate and mimicker of metastasis. Eur J Cancer. 2020 May;131:18-26.

4 Hendrickx BW, van Herpen CM, Bonenkamp JJ, Bulten J, Oyen WJ. Positive positron emission tomography scan in sarcoidosis and two challenging cases of metastatic cancer. CASE 1. Mediastinal sarcoidosis in a melanoma patient treated with interferon. J Clin Oncol. 2005 Dec 1;23(34):8906-7.

5 Koo HJ, Kim MY, Shin SY, Shin S, Kim SS, Lee SW, et al. Evaluation of mediastinal lymph nodes in sarcoidosis, sarcoid reaction, and malignant lymph nodes using CT and FDG-PET/CT. Medicine. 2015 Jul;94(27):e1095.

6 Spiekermann C, Kuhlencord M, Huss S, Rudack C, Weiss D. Coexistence of sarcoidosis and metastatic lesions: a diagnostic and therapeutic dilemma. Oncol Lett. 2017 Dec;14(6):7643-52.

7 Chaigne B, Perrinaud A, Penaud A, Machet MC, Venel Y, Marchand-Adam S, et al. Melanoma lymph node metastasis occurring simultaneously with multifocal sarcoidosis affecting lymph nodes and the lung: a diagnostic pitfall. Eur J Dermatol. 2011 Sep-0ct;21(5):798-9.

8 Malli F, Gourgoulianis KI, Daniil Z. Concurrent sarcoidosis and melanoma: coexistence more than by chance? Dermatology. 2010;221(4):320.

9 von Euw E, Chodon T, Attar N, Jalil J, Koya RC, Comin-Anduix B, et al. CTLA4 blockade increases Th17 cells in patients with metastatic melanoma. J Transl Med. 2009 May 20;7:35.

10 Braun NA, Celada LJ, Herazo-Maya JD, Abraham S, Shaginurova G, Sevin CM, et al. Blockade of the programmed death-1 pathway restores sarcoidosis CD4(+) T-cell proliferative capacity. Am J Respir Crit Care Med. 2014 Sep 1;190(5):560-71.

11 Rozeman EA, Menzies AM, van Akkooi ACJ, Adhikari C, Bierman C, van de Wiel BA, et al. Identification of the optimal combination dosing schedule of neoadjuvant ipilimumab plus nivolumab in macroscopic stage III melanoma (OpACIN-neo): a multicentre, phase 2, randomised, controlled trial. Lancet Oncol. 2019 Jul;20(7): 948-60. 
12 Eggermont AMM, Blank CU, Mandala M, Long GV, Atkinson V, Dalle S, et al. Adjuvant pembrolizumab versus placebo in resected stage III melanoma. N Engl J Med. 2018 May 10;378(19):1789-801.

13 Lheure C, Kramkimel N, Franck N, Laurent-Roussel S, Carlotti A, Queant A, et al. Sarcoidosis in patients treated with vemurafenib for metastatic melanoma: a paradoxical autoimmune activation. Dermatology. 2015; 231(4):378-84.

14 Adam A, Thomas L, Bories N, Zaharia D, Balme B, Freymond N, et al. Sarcoidosis associated with vemurafenib. Br J Dermatol. 2013 Jul;169(1):206-8.

15 Seve P, Schott AM, Pavic M, Broussolle C, Gilis L, Thomas L. Sarcoidosis and melanoma: a referral center study of 1,199 cases. Dermatology. 2009;219(1):25-31. 